\title{
Corrigendum: Dissipative Magnetic Soliton in a Spinor Polariton Bose-Einstein Condensate
}

\author{
Chunyu Jia ${ }^{1}$, Rukuan $\mathrm{Wu}^{1}$, Ying $\mathrm{Hu}^{2,3 *}$, Wu-Ming $\mathrm{Liu}^{4,5,6}$ and Zhaoxin Liang ${ }^{1 *}$ \\ ${ }^{1}$ Department of Physics, Zhejiang Normal University, Jinhua, China, ${ }^{2}$ State Key Laboratory of Quantum Optics and Quantum \\ Optics Devices, Institute of Laser Spectroscopy, Shanxi University, Taiyuan, China, ${ }^{3}$ Collaborative Innovation Center of Extreme \\ Optics, Shanxi University, Taiyuan, China, ${ }^{4}$ Beijing National Laboratory for Condensed Matter Physics, Institute of Physics, \\ Chinese Academy of Sciences, Beijing, China, ${ }^{5}$ School of Physical Sciences, University of Chinese Academy of Sciences, Beijing, \\ China, ${ }^{6}$ Songshan Lake Materials Laboratory, Dongguan, China
}

Keywords: exciton-polariton Bose-Einstein condensate, soliton, excitation, Bogoliubov-de Gennes equation, spinor

\section{OPEN ACCESS}

Approved by:

Frontiers Editorial Office,

Frontiers Media SA, Switzerland

${ }^{*}$ Correspondence: Ying $\mathrm{Hu}$

huying@sxu.edu.cn

Zhaoxin Liang

zhxliang@gmail.com

Specialty section:

This article was submitted to Optics and Photonics, a section of the journal

Frontiers in Physics

Received: 05 January 2022 Accepted: 06 January 2022 Published: 04 February 2022

Citation:

Jia C, Wu R, Hu Y, Liu W-M and

Liang Z (2022) Corrigendum:

Dissipative Magnetic Soliton in a

Spinor Polariton

Bose-Einstein Condensate.

Front. Phys. 10:848786.

doi: $10.3389 /$ fphy.2022.848786

\section{A corrigendum on}

Dissipative Magnetic Soliton in a Spinor Polariton Bose-Einstein Condensate

by Jia, C., Wu, R., Hu, Y., Liu, W.-M., and Liang, Z. (2021). Front. Phys. 9:805 841. doi: 10.338 9/fphy. 2021.805841

In the original article, the authors neglected to include the funder "National Natural Science Foundation of China, Grant No. 11975208" to the author RW in the Funding statement. The updated Funding statement can be found below.

The authors apologize for this error and state that this does not change the scientific conclusions of the article in any way. The original article has been updated.

\section{FUNDING}

The authors declare that they received funding from the National Natural Science Foundation of China, Grant No. 11975208 to the author RW.

Publisher's Note: All claims expressed in this article are solely those of the authors and do not necessarily represent those of their affiliated organizations, or those of the publisher, the editors and the reviewers. Any product that may be evaluated in this article, or claim that may be made by its manufacturer, is not guaranteed or endorsed by the publisher.

Copyright $\odot 2022 \mathrm{Jia}, \mathrm{Wu}, \mathrm{Hu}$, Liu and Liang. This is an open-access article distributed under the terms of the Creative Commons Attribution License (CC BY). The use, distribution or reproduction in other forums is permitted, provided the original author $(s)$ and the copyright owner(s) are credited and that the original publication in this journal is cited, in accordance with accepted academic practice. No use, distribution or reproduction is permitted which does not comply with these terms. 